\title{
Optimizing City Traffic Light Management for Improving Traffic System in Smart Cities
}

\author{
Md Ilyas \\ M.E. Scholar \\ 26 South Gafoor Khan Ki Bazariya Indore (M.P.) \\ India
}

\author{
Varsha Zokarkar \\ Reader, CSE (SDBCE) \\ 162, Lokmanya Nagar Indore (M.P.) India
}

\begin{abstract}
The network technology is growing continuously and every day a new and innovative research is performed for improving the communication cost and performance. Due to this a number of different applications are developed for supporting the human being for their daily usages. Among them the monitoring and surveillance is a key aspects of the different applications. In this presented work the key aim is to study about the different wireless network technologies and obtain an effective manner to use with the city traffic management systems. There a number of techniques are found when studied around the city traffic management system. Among them the VANET (vehicular ad hoc network) is one of the most cost effective and efficient network technologies which are frequently used for the traffic monitoring and surveillance. Therefore in this presented work the key focused is first placed on studying about the VANET networks and then after a RSU (road side unit based) VANET is identified to use with the city traffic network. In this technique V2R (vehicle to RSU) and V2I (vehicle to infrastructure) technique is used to simulate the network traffic issues and their signaling process. The main concept behind the presented simulation is that the scheduling the city traffic network by the traffic lights. This may help to improve the scheduling of the traffic lights to improve the traffic management. In addition of that a priority based scheduling algorithm is also developed for finding the better schedule of the vehicles during heavy traffic in roads. The implementation of the proposed functional demonstration is performed on the basis of SUMO tool. And the scheduling algorithm is implemented using the JAVA technology. After the simulation the performance of scheduling algorithm is estimated and demonstrated in terms of time complexity, space complexity and the decisional ability. The results demonstrate the effective scheduling of traffic lights and improved management of the city traffic.
\end{abstract}

\section{Keywords}

Urban traffic issues, traffic lights, VANET, RSU, traffic management.

\section{INTRODUCTION}

Increasing traffic in the country in urban areas leads unwanted accidents, traffic jam and others due to this a significant amount of health and wealth are lost. Therefore a new kind of traffic management technique is required to reduce the traffic loads on streets and also reduces the losses. In order to find the solution numbers of issues are encountered during the investigation of proper methodology of traffic management. Among different technologies the VANET (vehicular ad hoc network) promises to provide the end to end solution for traffic monitoring and prevention from the accidents [1]

Therefore the proposed work involved for configuring the vehicular ad hoc network for enhancing the traffic issues and traffic load on the streets additionally also required improving the communication methodology for priority based traffic management in traffic signalling. Basically the VANET is a kind of ad hoc network where the network nodes (vehicles) are communicating each other or infrastructure to gent advantage of management and surveillance services.

The wireless communication is advancing continuously and researches are continuously making efforts for finding the fruitful results from the wireless communication technology. A significant amount of applications are using this technology in different area of science, technology, research and life styles. Therefore that is an essential domain of research and application development. The wireless communication enables us to deploy the rapid configuring network, mobile nodes and ad hoc nature of services. Among various ad hoc network technologies the VANET is one of the most essential technologies for application development.

In this presented work a keen study on VANET technology is performed and their different kinds of new generation applications are explored. Among them the traffic management is a key domain of study using VANET and by improving the scheduling of the traffic various real world problems are resolved. Therefore the proposed work is further extended for the managing the traffic lights in city roads. In addition of that a service model is proposed using the weighted method or priority estimation of the vehicles for regulating the traffic lights and optimizing the optimum route discovery method.

This section provides the basic overview of the proposed model next section describes the methods used and assumed for demonstration.

\section{PROPOSED WORK}

According to the observations the urban traffic is not much regular and can be fluctuating in time varying in nature. Therefore in order to proper management an adoptive scheme is required which regulate the traffic according to the need of the current traffic loads. Therefore the following issues are considered to overcome during the proposed work.

1. Traffic light management according to the traffic load: most of the traffic signals are regulated on the basis of time thus even the traffic is cleared the other side vehicles are waiting. On the other hand the high traffic signals provide the small amount of time to clear the traffic thus that is required to optimize the mechanism for signaling.

2. Traffic jam conditions: during investigation that is found most of the traffic jams are occurred due to unscheduled traffic systems. The traffic lights are providing same slots of time for low traffic signals as the highly loaded singles that causes a huge traffic issues and a significant amount of high priority vehicles are waiting for the road Clearance. 
3. Path for high priority vehicles: in urban traffic the different kinds of vehicles are traveling in roads but some of them are running in critical situations. Therefore if the traffic lights are regulated in the time based manner these vehicles are not obtaining their destination as they are waited for. For example school buses, ambulance, fire control vehicles and others.

The key aim of the proposed system, to recover the traffic knowledge of the signals using advance network communication technology and provides the optimum schedule for distributing the traffic load. Therefore in first the network model is described by which the traffic information is extracted.

\section{Network modeling:}

In this work the VANET (vehicular ad hoc network) is utilized for the network development and the traffic information extraction. The entire network contains three different kinds of devices all the used devices are described as:

1. Vehicle nodes: vehicles are the WI-FI devices and usage only wireless communication. In order to participate with the traffic management technique using VANET that is required to each the vehicle are mounted with the Wi-Fi devices to communicate each other and at the same time with the installed RSUs (road side units).

2. RSU nodes: RSU (road side units) are the bi-directional communication devices which is first works as the Wi-Fi to communicate with the vehicles. And in second the RSUs are connected with the dedicated backbone networks. That is a kind of high speed network to deliver the traffic samples to the server system and get the information from the server system to distribute it to the network.

3. Decision making server: that is a high performance computing machine which find the network from the entire city and aggregated on this machines database. That is further processed for extracting the knowledge of the city traffic and loads on the traffic signals. According to the generated traffic on the signals the decisions of traffic light switching is distributed.

\section{Network management}

The entire city contains a significant amount of vehicles and in similar ways a good quality of RSUs. But need to pass the instructions by identifying the targeted square to deliver the decisions for switching the traffic signals. Therefore each square in the city is addressed by a unique identifier and their ID is preserved in the server system. After executing the traffic samples over the decision server the instructions are forwarded to the RSU placed in the specific square ID. And using the RSU instructions the traffic lights are regulated.

\section{Priority based algorithm}

In order to demonstrate the weighted scheduling technique for the traffic load distribution some additional assumptions are required to place. Therefore some of the priority vehicle is prepared and reported using the table 1 .
Table 1 priority settings

\begin{tabular}{|c|c|c|}
\hline S. no. & Vehicle & Priority \\
\hline 1 & Fire vehicle & 1.0 \\
\hline 2 & School vehicle & 0.5 \\
\hline 3 & Normal vehicle & 0.1 \\
\hline
\end{tabular}

The given priority of vehicles is aggregated at the time of decision making for aggregating the weights of the traffic load the following formula is used

$$
W=\sum_{i=1}^{N} V_{p}
$$

Where,

$\mathrm{W}=$ the traffic weight of a direction

$\mathrm{N}=$ number of vehicles at time $T_{1}$

$V_{p}=$ the priority of vehicle

In this case the $V_{p}$ can be used with the following conditions

$$
V_{p}=\left\{\begin{array}{l}
1.0 \quad \text { if } p=\text { high } \\
0.5 \text { if } p=\text { avarage } \\
0.1 \quad \text { if } p=\text { low }
\end{array}\right.
$$

In order to compute and decide the switching of traffic signal the following procedure is taken place:

Suppose there $\mathrm{N}$ vehicle arrived in different directions in the traffic signals and there are D dimensions to distribute the traffic thus

1. For $(i=1 ; i<=D ; i++)$

a. Compute the weights from first sample of time $T_{1}$

b. If $w_{i}>w_{i+1}$ then

i. Trigger the lights for $i^{\text {th }}$ direction

ii. Wait till all the vehicles at time $T_{1}$ passed through

c. End if

\section{End for}

This section provides the understanding of the proposed working model and simulation aspect for the traffic light management and the required weighted algorithm for schedule the traffic signals.

\section{RESULT ANALYSIS}

In this section the implemented simulation is evaluated on the different performance parameters. The obtained results in different conditions are evaluated and demonstrated and compared with the normal configured traffic simulation using the graphical representation.

\subsection{Time complexity}

The amount of time required to compute the schedule the traffic light required to execute a designed algorithm thus the time of execution is given as the time complexity and demonstrated figure 1 . 


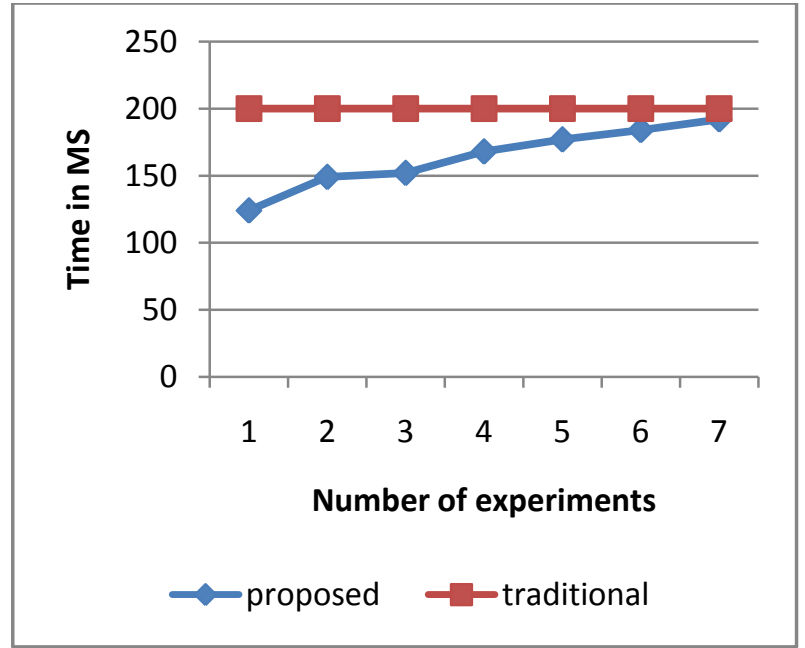

Fig 1 time complexity

In this given figure 1 the time complexity of both the techniques are demonstrated. The time based scheduling is given using red line which is not fluctuating and remains constant because the traffic lights are scheduled according to the given time slots. But the proposed techniques lights are scheduled according to the arrived traffic over the traffic signals. The time complexity of the proposed technique is demonstrated using the blue line. According to the given results the proposed technique consumes the less amount of time as compared to the traditional technique. In this diagram the $\mathrm{X}$ axis shows the different experiments performed with the implemented simulation with the increasing traffic load on singles and the $\mathrm{Y}$ axis shows amount of time required to schedule in terms of milliseconds. In further the performance in tabular manner is given using table 2 .

Table 2 time complexity

\begin{tabular}{|c|c|c|}
\hline $\begin{array}{c}\text { Number of } \\
\text { experiments }\end{array}$ & $\begin{array}{c}\text { Proposed } \\
\text { technique }\end{array}$ & $\begin{array}{c}\text { Traditional } \\
\text { method }\end{array}$ \\
\hline 1 & 124 & 200 \\
\hline 2 & 149 & 200 \\
\hline 3 & 152 & 200 \\
\hline 4 & 168 & 200 \\
\hline 5 & 177 & 200 \\
\hline 6 & 184 & 200 \\
\hline 7 & 192 & 200 \\
\hline
\end{tabular}

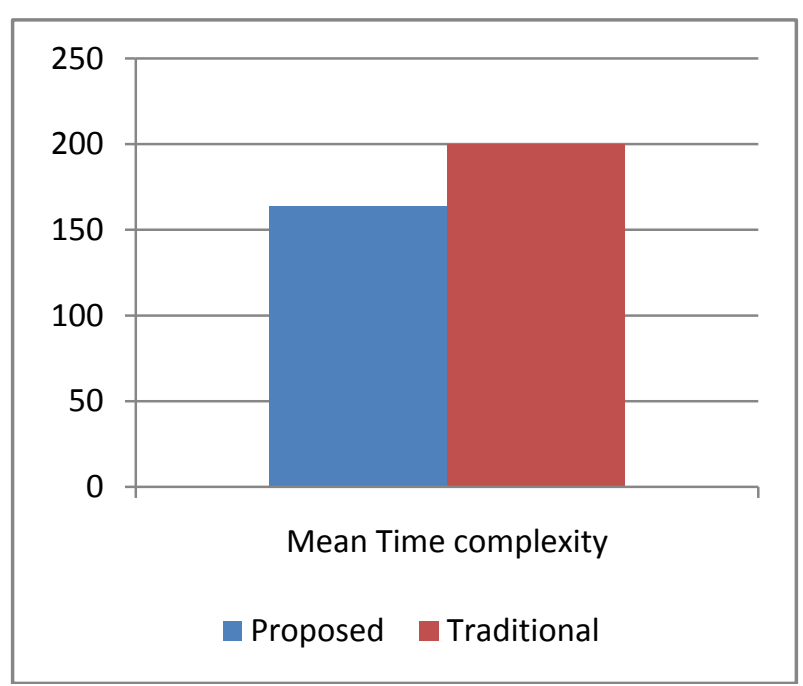

Fig 2 mean time complexity

In order to refine the results and provides the clear justification of time complexity of the scheduling time of the traffic lights, the mean time complexity is computed through the different experiments performed. The mean time complexity of both the algorithms is described in the figure 2 . In this diagram the $X$ axis contains the different techniques that are implemented and the $\mathrm{Y}$ axis shows the mean time complexity of the algorithms in terms of milliseconds. According to the obtained mean time consumption, the proposed technique is much efficient as compared to the traditional technique.

\subsection{Space complexity}

The amount of main memory required to execute the traffic light scheduling algorithm is given as the space complexity. The space complexity of both the implemented techniques are given using the figure 3 in this diagram the red line shows the performance of traditional approach and the blue line shows the performance of the proposed algorithm. In order to represent the performance of the algorithms the $\mathrm{X}$ axis shows the different number of experiments performed with the system with increasing traffic load and the $\mathrm{Y}$ axis shows the amount of memory consumed to process the traffic load.

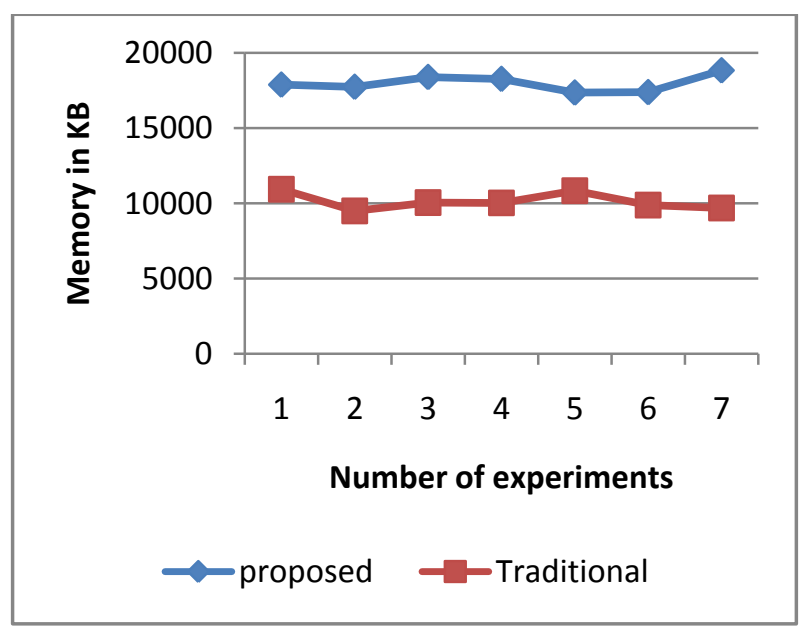

Fig 3 space complexity

According to the given experimental results the traditional technique shows the less memory consumption as compared 
to the traditional technique. Because the traditional technique not executes the any algorithm to schedule the traffic singles that only switch the signals according to the time based fashion. The given table 3 shows the performance of the algorithms in terms of memory consumption.

Table 3 memory consumption

\begin{tabular}{|c|c|c|}
\hline $\begin{array}{c}\text { Number of } \\
\text { experiments }\end{array}$ & $\begin{array}{c}\text { Proposed } \\
\text { technique }\end{array}$ & $\begin{array}{c}\text { Traditional } \\
\text { technique }\end{array}$ \\
\hline 1 & 17883 & 10937 \\
\hline 2 & 17739 & 9488 \\
\hline 3 & 18392 & 10048 \\
\hline 4 & 18266 & 10023 \\
\hline 5 & 17355 & 10833 \\
\hline 6 & 17383 & 9878 \\
\hline 7 & 18837 & 9682 \\
\hline
\end{tabular}

In order to more clarify the difference among the proposed and traditional technique the figure 4 shows the mean memory consumption of the algorithms. In this diagram the $\mathrm{X}$ axis shows the different techniques implemented and the $\mathrm{Y}$ axis shows the performance of technique in terms of memory consumption. According to the obtained memory consumption the proposed technique consumes more memory as compared to the traditional technique. Thus according to the memory consumption the proposed technique is leaked in performance.

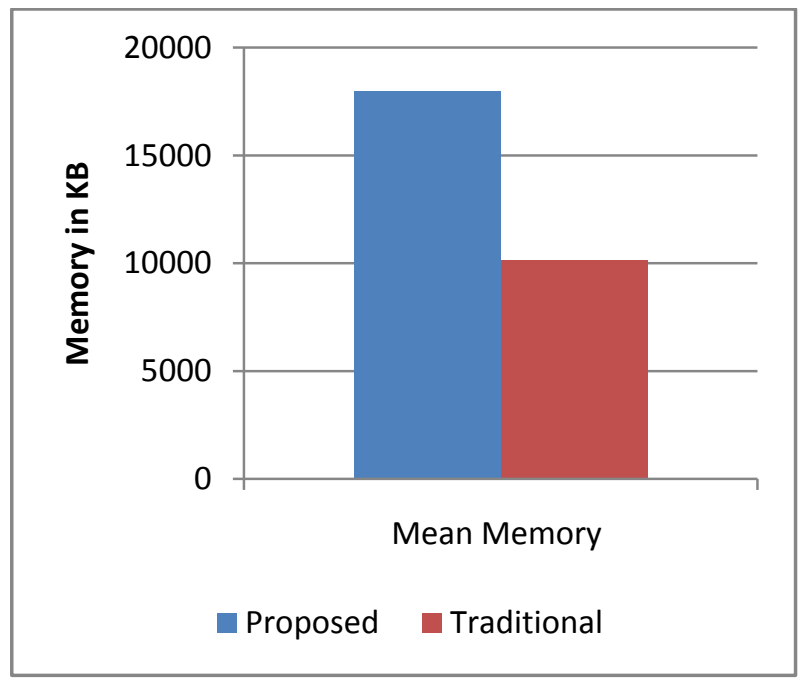

Fig 4 mean memory consumption

\subsection{Traffic light decision time}

Sometimes the load on a signal is low and on the other part of signal is high thus for effective scheduling required to regular and uniform switching of the traffic lights according to the traffic load on signals. The decision time of traffic signal demonstrate how frequently a signal is switched during high load and low traffic load on any signal. The given table 4 shows the traffic scheduling during the high load scenarios and similarly during the low traffic signals how the traffic lights are switched is given using table 5. Correspondingly the figure 5 shows the high traffic scenario and figure 6 shows the low traffic scenario results. In order to demonstrate the results of both the scenarios the $\mathrm{X}$ axis of the figure provides the different number of experiments performed and the $\mathrm{Y}$ axis shows the amount of time required to schedules. According to the obtained results during the high load conditions the lights are switched in more time as compared to low traffic conditions in the proposed techniques but during the normal conditions that remains fix and low traffic is cleared during the high load conditions.

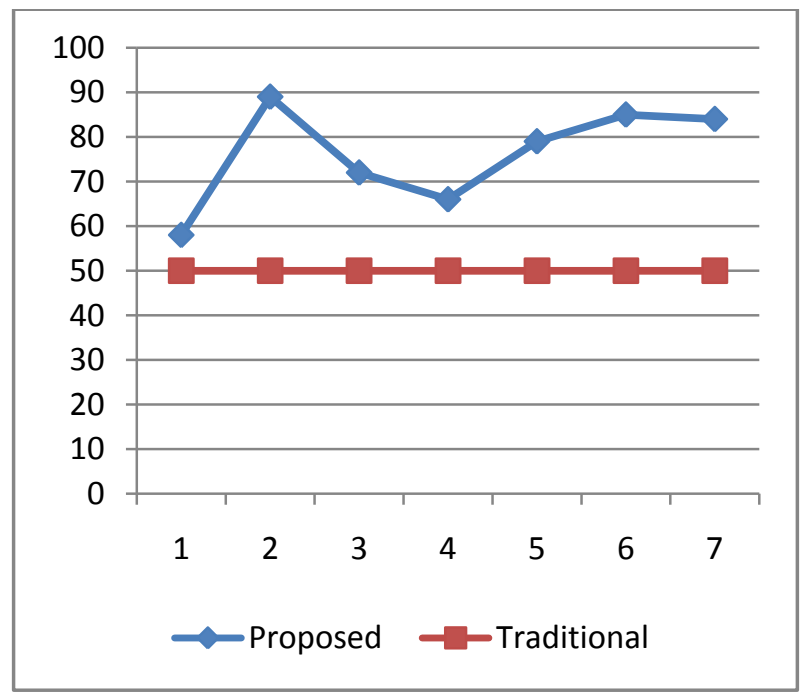

Fig 5 high traffic

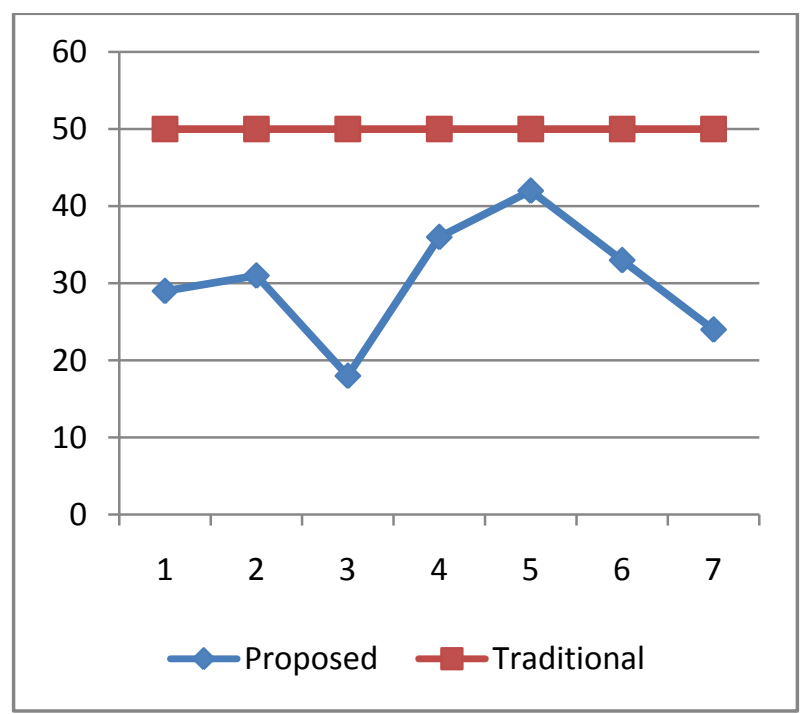

Fig 6 low traffic

Table 4 high traffic conditions

\begin{tabular}{|c|c|c|}
\hline $\begin{array}{c}\text { Number of } \\
\text { experiments }\end{array}$ & $\begin{array}{c}\text { Proposed } \\
\text { technique }\end{array}$ & $\begin{array}{c}\text { Traditional } \\
\text { technique }\end{array}$ \\
\hline 1 & 58 & 50 \\
\hline 2 & 89 & 50 \\
\hline 3 & 72 & 50 \\
\hline
\end{tabular}




\begin{tabular}{|c|c|c|}
\hline 4 & 66 & 50 \\
\hline 5 & 79 & 50 \\
\hline 6 & 85 & 50 \\
\hline 7 & 84 & 50 \\
\hline
\end{tabular}

Table 5 low traffic

\begin{tabular}{|c|c|c|}
\hline $\begin{array}{c}\text { Number of } \\
\text { experiments }\end{array}$ & $\begin{array}{c}\text { Proposed } \\
\text { technique }\end{array}$ & $\begin{array}{c}\text { Traditional } \\
\text { technique }\end{array}$ \\
\hline 1 & 29 & 50 \\
\hline 2 & 31 & 50 \\
\hline 3 & 18 & 50 \\
\hline 4 & 36 & 50 \\
\hline 5 & 42 & 50 \\
\hline 6 & 33 & 50 \\
\hline 7 & 24 & 50 \\
\hline
\end{tabular}

According to the obtained decisional results the decision time for both the conditions are summarized using figure 7 in this diagram the low traffic and high traffic conditions are demonstrated using $\mathrm{X}$ axis and the $\mathrm{Y}$ axis contains the corresponding mean time of light switching.

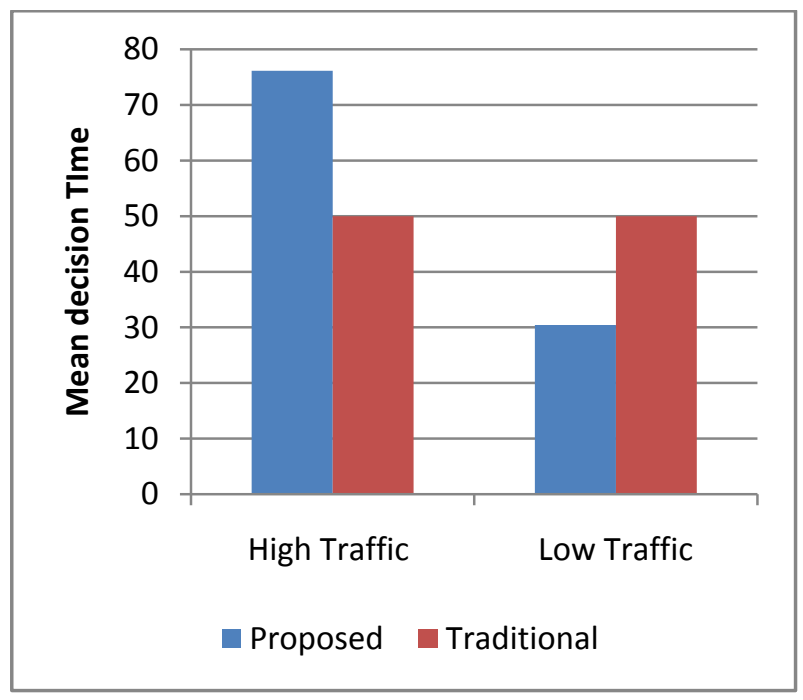

Fig 7 traffic decision time

\section{CONCLUSIONS}

The proposed work is intended to find the solution for traffic light management for reducing the traffic load on urban area. Using the experimentation and the observation a weighted algorithm is proposed and the summary of system design is reported in this chapter. In addition of that the future extension of the work is also suggested.

\subsection{Conclusion}

Indian is a developing country and due to this a huge population is reside in India. Additionally a significant amount of peoples are moving towards the cities and due to this a huge crowed is appeared on the city streets. Therefore the urban road traffic is also affected in day by day fashion. In this presented work the study is made on the traffic of urban areas and that is observed if the traffic lights are scheduled in efficient manner than the load on the city streets are becomes less. This reduces the efforts of traffic management staff and also reduces the delay on traffic directions.

With the aim of efficient and effective traffic management system the VANET (vehicular ad hoc networks) techniques of traffic simulation is studied. The VANET is one of the most popular network technologies for providing ease in traffic management. The VANET is not only used with the traffic management system that having a broad area of different applications. In this presented system the VANET is considered with the RSUs (road side units). These road side units are connected together with the backbone network and used to collect the traffic information from the vehicles. The collected information is transmitted to the decision making server which collect the entire the network information from the network and process them to provide ease in traffic management.

The implementation of the simulation of VANET technique performed with the help of SUMO tool. That enables us to use the different traffic scenario and different kinds of vehicles for utilizing them. Additionally a JAVA based weighted algorithm is prepared to generate the traffic light switching information for the traffic signals. The implementation and design of the proposed concept of the traffic simulation is performed more effectively and their impacts on the different kinds of traffic loads are observed. In order to justify the proposed solution the implemented technique is compared with the traditional technique of traffic management where the traffic signals are regulated using the time based fashion. According to the obtained results and evaluated parameters the performance of both the implemented conditions are summarized using the table 5 .

Table 5 performance summary

\begin{tabular}{|c|c|c|c|}
\hline $\begin{array}{c}\text { S. } \\
\text { No. }\end{array}$ & Parameters & Proposed & Traditional \\
\hline 1 & Time complexity & Efficient & Constant \\
\hline 2 & Space complexity & Poor & Efficient \\
\hline 3 & Decision time & Efficient & Constant \\
\hline
\end{tabular}

According to the obtained results the proposed technique is outperform for the traffic signal management and their switching. That makes and impressive decisions during the high traffic load and low traffic load in both the conditions. On the other hand as compared to the traditional method the memory consumption of the proposed technique is higher than the traditional approach of time based scheduling.

\subsection{Future extension}

In this presented work the key objective of the traffic light management is accomplished using the VANET technology. In near future the proposed technique is implemented for navigation and shortest path finding technique for dense 
traffic management. Additionally the use of the proposed weighted technique is provided for finding the optimum path that consumes less time as compared to the shortest path evaluation.

\section{REFERENCES}

[1] Rotterdam, "Study on Urban Aspects of the Internalisation of External Costs", November 2012, http://ec.europa.eu/transport/themes/urban/studies/doc/20 12-study-on-urban-aspects-of-the-internalisation-ofexternal-costs.pdf

[2] Rahul Goyal, Ms. ShraddhaMasih, "Cloudlet Assisted Mobile Cloud Computing Environment For Traffic Management", School of Computer Science \& IT, (University Teaching Department), Devi Ahilya University, Indore (M.P), JUNE 2013

[3] Balmahoon R, Peplow R, "Vehicular Ad-Hoc Networks: An Introduction to Privacy",Southern African Telecommunication Networks and Applications Conference (SATNAC) will be held from 2 to 5 September 2012

[4] Maen M Artimy, William Robertson, and William J Phillips, Algorithms and Protocols for Wireless and Mobile Ad Hoc Networks, 1st ed., AzzedineBoukerche, Ed.: John Wiley \&Sons, 2009.

[5] S. A. Ade \& P. A. Tijare, "Performance Comparison of AODV, DSDV, OLSR and DSR Routing Protocols in Mobile Ad Hoc Networks", International Journal of Information Technology and Knowledge Management, July-December 2010, Volume 2, No. 2, pp. 545-548

[6] AmeurBennaoui, Mustapha Guezouri and MokhtarKeche, "Performance Evaluation of Routing Protocols in Vehicular Networks", I. J. Computer Network and Information Security, 2013, 10, 11-16, Published Online August 2013 in MECS

[7] S.Sujatha, P.Sukanya, "Multicast Dual Arti-Q System in Vehicular Adhoc Networks", IOSR Journal of Computer Engineering (IOSR-JCE), e-ISSN: 2278-0661, p- ISSN: 2278-8727Volume 12, Issue 3 (Jul. - Aug. 2013), PP 9095

[8] ZaydounYahyaRawashdeh and Syed Masud Mahmud, "Communications in Vehicular Networks", Wayne State University, Detroit, Michigan, USA, http://www.intechopen.com/

[9] Peter Lerchbaumer and Alejandro Ochoa, "Test Environment Design for Wireless Vehicle Communications", Master's Thesis in Computer Systems Engineering, Technical report, IDE0710, January 2007

[10] Christophe J. Merlin and Wendi B. Heinzelman, "A Study of Safety Applications in Vehicular Networks", Manuscript received July 1st, 2005. http://www.ece.rochester.edu/

[11] IliasLeontiadis, Gustavo Marfia, David Mack, Giovanni Pau, Cecilia Mascolo, and Mario Gerla, "On the Effectiveness of an Opportunistic Traffic Management
System for Vehicular Networks", IEEE Transactions on Intelligent Transportation Systems, Vol. 12, No. 4, December 2011

[12] Katrina Chapman, "System Support for Operators in a Traffic ManagementControl Centre", 19th ITS World Congress, Vienna, Austria, 22/26 October 2012

[13] Monika Singh, RuhiSaxena, "An Application of UML for Road Traffic Management System by Implementing Extensive Mechanism: Stereotypes", IJCSI International Journal of Computer Science Issues, Vol. 11, Issue 5, No 1, September 2014

[14] Habib M. Kammoun, IlhemKallel, Jorge Casillas, Ajith Abraham, Adel M. Alimi, "Adapt-Traf: An adaptive multiagent road traffic management system based on hybrid ant-hierarchical fuzzy model”, 2014 Elsevier Ltd. All rights reserved.

[15] Dongbin Zhao, Yujie Dai, and Zhen Zhang, "Computational Intelligence in Urban Traffic Signal Control: A Survey", IEEE Transections on Systems, Man, And Cybernetics-Part C: Applications And Reviews, Vol. 42, No. 4, July 2012

[16] Vicente Milanés, Jorge Villagrá, Jorge Godoy, Javier Simó, Joshué Pérez, and Enrique Onieva, "An Intelligent V2I-Based Traffic Management System”, IEEE Transactions on Intelligent Transportation Systems, Vol. 13, No. 1, March 2012

[17] Gabriel Kapapula, Pardeep Mittal, "A Novel Approach for Dynamic Road Traffic Management System (DRTMS) with Lab VIEW and Arduino", International Research Journal of Engineering and Technology (IRJET), Volume: 02 Issue: 05 | Aug-2015

[18] SheraliZeadally, Ray Hunt, Yuh-Shyan Chen, Angela Irwin, Aamir Hassan, "Vehicular ad hoc networks (VANETS): status, results, and challenges", (C) Springer Science+Business Media, LLC 2010.

[19] Javier Barrachina, PiedadGarrido, Manuel Fogue, Francisco J. Martinez, Juan-Carlos Cano, Carlos T. Calafate, Pietro Manzoni, "CAOVA: A Car Accident Ontology for VANETs", 2012 IEEE Wireless Communications and Networking Conference: Mobile and Wireless Networks

[20] Miao Wang, Hangguan Shan, Rongxing Lu, Ran Zhang, Xuemin (Sherman) Shen, and Fan Bai, "Real-Time Path Planning Based on Hybrid-VANET-Enhanced Transportation System", IEEE Transactions on Vehicular Technology, Vol. 64, No. 5, May 2015

[21] Estrella Garcia-Lozano, Carolina Tripp Barba, Monica Aguilar Igartua and Celeste Campo, "A distributed, bandwidth-efficient accident prevention system for interurban VANETs", 2013 International Conference on Smart Communications in Network Technologies $(\mathrm{SaCoNeT})$ 